\title{
Trastornos del ánimo, psicofármacos y tiroides
}

\author{
Danilo Quiroz $\mathbf{L}^{\mathbf{1}}$, Sergio Gloger $\mathbf{K}^{\mathbf{1}}$, Sergio Valdivieso $\mathbf{F}^{\mathbf{2}}$, \\ José Ivelic $\mathrm{Z}^{2 \mathrm{a}}$, Carlos Fardella B ${ }^{3}$. \\ Mood disorders, \\ psychopharmacology and thyroid \\ hormones
}

For more than 40 years thyroid hormones and mood disorders have been associated. Some psychiatric symptoms are produced by thyroid illnesses and there is a frequent association of thyroid dysfunction with mood disorders. Therefore, routine thyroid function assessment in patients with mood disorders and the treatment of sub-clinical thyroid dysfunctions is recommended. The usefulness of adding thyroid hormones to antidepressive treatment in euthyroid patients to obtain a potentiation effect has been probed repeatedly. The most common strategy is potentiation with T3, but high doses of T4 have been also used in patients with resistant depression. Thyroid hormones exert their action in the central nervous system through a variety of mechanisms: modulation of gene expression of several groups of proteins, some of them with known physiopathological implications in mood disorders and the influence over serotonin and noradrenergic neurotransmission, known to be one of the modes of action of antidepressants. Finally, it is also important to stress the complex relationship between psychiatric drugs, deiodinases and thyroid hormones, that can potentially help to understand the mechanisms of action of these drugs (Rev Méd Chile 2004; 132: 1413-24).

(Key Words: Mood disorders; Psychopharmacology)

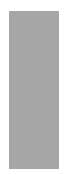

Recibido el 4 de marzo, 2004. Aceptado en versión corregida el 4 de agosto, 2004.

${ }^{1}$ Centro de Investigaciones Clínicas PsicoMédica. ${ }^{2}$ Departamento de Psiquiatría, ${ }^{3}$ Departamento de Endocrinología, Facultad de Medicina, Pontificia Universidad Católica de Chile. Santiago de Chile.

aResidente de Psiquiatría

L a indemnidad funcional del eje hipotálamohipófisis-tiroides, es fundamental para producir la hormona tiroidea necesaria para mantener el normal funcionamiento de prácticamente todos los órganos de la economía ${ }^{1}$. Mención especial

Correspondencia a: Dr. Carlos Fardella. Departamento de Endocrinología, Facultad de Medicina, Pontificia Universidad Católica de Chile, Lira 85, Santiago, Chile. Fax: 6385675. E mail: cfardella@med.puc.cl merece el sistema nervioso central, ya que una disfunción del eje puede generar sintomatología similar a las enfermedades de la esfera neuropsiquiátrica. Tanto el hipo como el hipertiroidismo se manifiestan con síntomas muy parecidos a una enfermedad psiquiátrica. En el hipotiroidismo son frecuentes la astenia psicomotora, el letargo, la somnolencia, la pérdida de memoria y capacidad de concentración, que también son frecuentes en pacientes con episodios depresivos. Por otra 
parte, el hipertiroidismo puede ser causa de hiperactividad, taquicardia, nerviosismo, crisis vasomotora, irritabilidad y cambios de conducta, que pueden semejar crisis de pánico o episodios de hipomanía y manía (euforia) ${ }^{2}$ (Tabla 1 ).

La ausencia de síntomas patognomónicos que orienten al clínico en el diagnóstico diferencial entre enfermedad tiroidea y un trastorno psiquiátrico, debiera hacer rutinaria la evaluación de función tiroidea en todo paciente que consulta por patología emocional. Esta sugerencia se basa en una reciente comunicación de nuestro grupo, que evaluó, en forma sistemática, la función tiroidea de consultantes a un centro de atención psiquiátrica ambulatoria ${ }^{3}$. Ese estudio, realizado en población chilena, demostró una alta prevalencia de hipotiroidismo en consultantes por episodios de depresión y crisis de pánico, superior a $10 \%$ de la muestra, afectando principalmente a mujeres en edad laboral. Reportes de otros países comunican frecuencias similares, en un rango entre 8 y 17\% de hipotiroidismo en pacientes con episodios depresivos ${ }^{4,5}$. La mayor parte de estos cuadros corresponden a hipotiroidismo subclínico, el cual se caracteriza por la ausencia o escasos signos clásicos de hipofunción tiroidea, niveles de hormonas tiroideas normales con hormona estimuladora de tiroides (TSH) en el límite superior o inmediatamente por sobre la norma ${ }^{6}$ y molestias anímicas como principal manifestación $n^{4,5,7,8}$.

La asociación entre disfunción tiroidea y patología emocional se incrementa cuando se evalúa a sujetos que han experimentado una pobre o nula respuesta a tratamiento psicofarmacológico. En nuestra casuística, el diagnóstico de hipotiroidismo llegó a duplicarse en relación a controles, alcanzando cifras cercanas a $25 \%$ de la muestra ${ }^{9}$. Una experiencia similar fue comunicada por Howland, quien encontró evidencias de hipotiroidismo en $52 \%$ de pacientes con depresión refractaria $^{8}$. La muy alta frecuencia de disfunción tiroidea en pacientes refractarios a tratamiento con psicofármacos, o que recaen después de una buena respuesta inicial, debiera hacer mandatorio evaluar o reevaluar su función tiroidea.

La importancia del diagnóstico precoz de una disfunción tiroidea radica en el positivo impacto que suele tener el tratamiento de ésta sobre la evolución del cuadro anímico. Asimismo, puede llevar aparejada una disminución en el número y dosis de psicofármacos utilizados, una mejoría de los parámetros lipídicos y de potenciales alteraciones cardiovasculares u otras anomalías que también suelen estar asociadas a la enfermedad tiroidea, tanto clínica como subclínica ${ }^{6}$. En pacientes que no presentan una disfunción tiroidea actual, la presencia de bocio o títulos elevados de anticuerpos antitiroideos debe considerarse un elemento importante en la identificación de sujetos en riesgo de desarrollarla y su pesquisa debiera incluirse en la evaluación inicial de cada paciente $^{9}$. Los autores sugieren la determinación de TSH ultrasensible, hormonas tiroideas y anticuerpos antitiroideos, las cuales debieran repetirse periódicamente en el seguimiento de pacientes en riesgo de desarrollar enfermedad tiroidea. Entre

Tabla 1. M anifestaciones neuropsiquiátricas asociadas a disfunción tiroidea

\begin{tabular}{|ll|}
\hline Hipertiroidismo & Hipotiroidismo \\
\hline Nerviosismo, ansiedad & Fatiga, letargia psicomotora \\
Labilidad emocional, angustia & Somnolencia progresiva \\
Hiperactividad psicomotora & Pérdida de memoria \\
Cambios de carácter & Pérdida de concentración \\
Pérdida de concentración & Dificultad para realizar cálculos \\
Disminución de la líbido & Apatía e indiferencia \\
Taquicardia, arritmias & Disminución de la líbido \\
Crisis vasomotoras & Disminución del apetito \\
Depresión en ancianos & Intolerancia al frío \\
Hipomanía o manía & Depresión \\
& Hipomanía o manía \\
\end{tabular}


estos últimos se incluyen aquellos que presentan antecedentes de enfermedad tiroidea familiar, episodios depresivos refractarios o aquellos que usan determinados psicofármacos (ver secciones posteriores), u otras drogas, como amiodarona, con reconocida acción tiroidea.

La presente revisión pretende orientar respecto del uso de hormonas tiroideas en pacientes con trastornos del ánimo, hipotiroidismo subclínico, actualizar la relación entre hormonas tiroideas y sistema nervioso central (SNC), y revisar las interacciones entre psicofármacos y función tiroidea.

\section{Uso De hormona tiRoidea EN PSiquiatría}

El uso de hormona tiroidea en pacientes con trastornos del ánimo está indicado en los portadores de una alteración tiroidea, o bien como acelerador o potenciador del tratamiento farmacológico en pacientes refractarios o que responden parcialmente a tratamiento y con niveles hormonales en rango normal.

Uso de hormona tiroidea en pacientes hipotiroideos. En estos pacientes se ha demostrado que el tratamiento debe seguirse bajo los conceptos clásicos que apuntan a la administración de tetraiodotironina (T4) en dosis suficientes para normalizar TSH. Sin embargo, la normalización de TSH debiera seguir criterios más estrictos que aquellos usados en sujetos sin patología psiquiátrica. En la actualidad se acepta la propuesta hecha por Haggerty ${ }^{10}$, que postula mantener los pacientes en que coexisten enfermedad tiroidea y un trastorno psiquiátrico con niveles de TSH por debajo del nivel de 3,0 $\mu \mathrm{U} / \mathrm{ml}$ y no de $5,0 \mu \mathrm{U} / \mathrm{ml}$, como se propone en población libre de patología psiquiátrica ${ }^{10,11}$. Se postula que en las primeras etapas del hipotiroidismo el sistema nervioso central sería más sensible que los órganos periféricos, dando manifestaciones más precoces y reversibles con el uso de hormona tiroidea.

El uso de terapia combinada de triiodotironina (T3 + T4) ha sido sugerida por algunos autores $^{12,13}$, pero no parece tener ventajas sobre el uso de monoterapia con $\mathrm{T} 4$ en el control de los síntomas ansiosos, depresivos o cognitivos ${ }^{14,15}$. Además, la sobredosis de T3 puede adicionar riesgos al tratamiento, como la pérdida de masa ósea, lo cual puede resultar especialmente deletéreo en mujeres posmenopáusicas que ya pueden tener algún grado de disminución de su contenido mineral óseo. Un grupo de pacientes más sensible al uso de hormonas tiroideas son aquellos que padecen de patología cardiovascular, en particular aquellos que acusan una cardiopatía coronaria 0 arritmias previas al inicio de terapia. También es importante considerar que en algunos pacientes, en sobredosis, la hormona tiroidea puede provocar disfunción hepática con elevación de transaminasas. Finalmente, también está descrito que en enfermos con depresión bipolar el uso de T3 puede desencadenar episodios maníacos ${ }^{16,17}$. Sin embargo, de acuerdo a la experiencia de los autores, el uso de T3 puede ser de utilidad en sujetos hipotiroideos que, una vez tratados con T4, no modifican sus valores basales de T3, aun después de normalizado el valor de TSH; en ellos se postula que existiría una falla en la conversión periférica de T4 a T3 (ver secciones siguientes y Figura 1) y dado que T3, y no T4, es el modulador final de la respuesta central a hormonas tiroideas es que estaría justificado su uso. La experiencia de

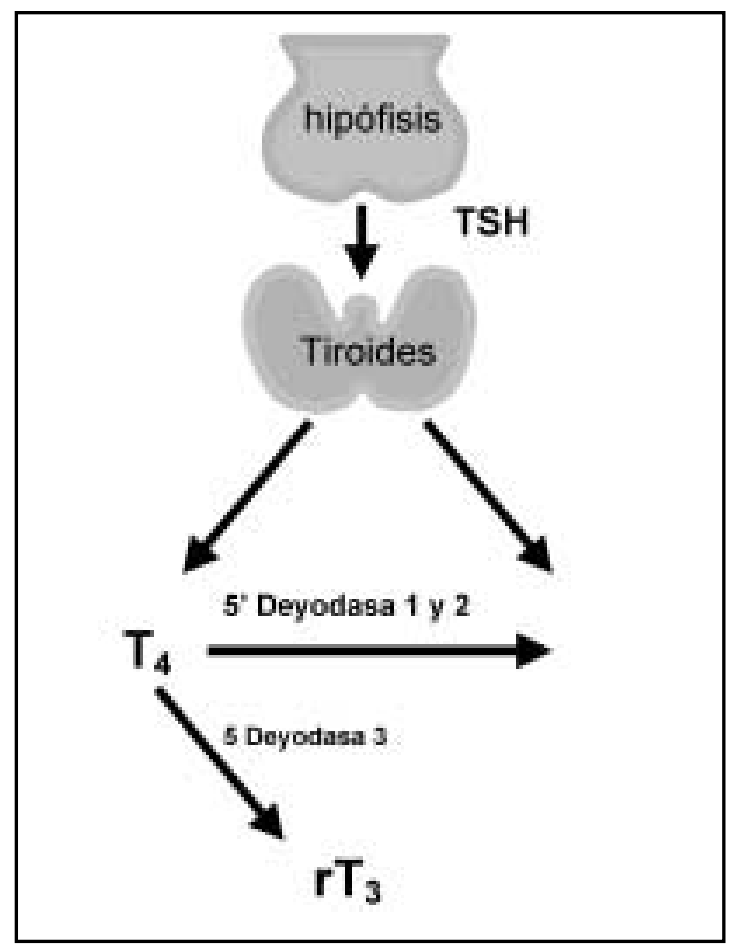

FIgURA 1. Metabolismo de la hormona tiroidea. 
los autores es que, en este grupo particular de pacientes, la adición de dosis bajas de T3 a la terapia con $\mathrm{T} 4$, promueve una notoria y continua mejoría anímica. En estos casos, la administración de T3 debe realizarse en forma fraccionada, ya que por su vida media corta (aproximadamente 8 h) produce picos en la sangre que obligan al uso de dosis pequeñas en, al menos, 2 tomas diarias.

Uso de hormona tiroidea en pacientes sin enfermedad tiroidea. En estos casos, la hormona tiroidea se usa como un acelerador de la respuesta a psicofármacos ${ }^{18-21}$, que consiste en el uso conjunto con un antidepresivo para promover una respuesta más rápida, o como potenciador, que consiste en adicionar hormona tiroidea a un paciente en tratamiento que ha respondido de manera parcial o nula $22-26$.

La utilidad de administrar T3 en pacientes deprimidos sin enfermedad tiroidea, está apoyada por estudios doble ciego, controlados por placebo, potenciando la utilización de antidepresivos tricíclicos (ej: amitriptilina, imipramina) y con estudios no controlados para inhibidores selectivos de la recaptura de serotonina (ej: fluoxetina, sertralina) e inhibidores de la monoaminoxidasa (IMAO). Para este uso se recomienda T3 en dosis de 25 a $50 \mu \mathrm{g} /$ día, por plazos de prueba de 2 a 4 semanas. Durante el tratamiento se debe observar la posible aparición de eventos adversos mencionados en la sección previa. El tiempo de mantención de la potenciación una vez obtenida la respuesta no es claro, sugiriéndose no suspenderlo mientras se mantenga el antidepresivo.

Otro uso de T3, administrado en dosis similares a las descritas, es el de acelerador de la respuesta antidepresiva. Nuevamente, los estudios controlados existentes son con antidepresivos tricíclicos ${ }^{20}$, por lo que falta aún evidencia más concluyente con nuevos antidepresivos. Cabe destacar que la mejor respuesta en esta indicación se observa en mujeres, a diferencia de la potenciación en que no se describe mejor respuesta por $\operatorname{sexo}^{26}$. Otro uso interesante de mencionar, es la combinación de T3 con terapia electroconvulsivante (TEC), con resultados alentadores en la respuesta antidepresiva y en menores efectos cognitivos de la TEC 27,28 .

Respecto del uso de T4, existe una serie de estudios abiertos que demuestran que dosis suprafi- siológicas de T4 (250-600 $\mu \mathrm{g} /$ día), son efectivas y bien toleradas cuando se agregan al tratamiento con antidepresivos o estabilizadores del ánimo, en pacientes con enfermedad afectiva monopolar o bipolar 29-31. Un hecho interesante de resaltar es la muy escasa o nula aparición de efectos adversos con estas dosis, a diferencia de lo que ocurre en pacientes con enfermedad timidea clásica. Así, los eventos cardiovasculares reportados son escasos y los signos de hipertiroidismo están ausentes en la mayor parte de ellos ${ }^{29,30,32}$, incluso con el uso a largo plazo ${ }^{33}$. Más aún, la pérdida de masa ósea no se verifica, y existen estudios que demuestran una ganancia en la densidad mineral ósea de pacientes tratados bajo este esquema ${ }^{33,34}$. La razón de esta respuesta sui géneris, que permite aliviar los síntomas depresivos sin que aparezcan efectos adversos, no es bien comprendida. Una posible explicación es que estos pacientes presenten una resistencia parcial a hormonas tiroideas, la cual sería posible superar sólo con el uso de macrodosis de esta hormona. Esta teoría se apoya en estudios en ratas sgenéticamente depresivas» que mejoran la conducta depresiva con el uso de macrodosis de hormona tiroidea, lo cual ha sido interpretado como secundario a una disminución de la sensibilidad a la acción de hormonas tiroideas ${ }^{35}$ (ver sección posterior).

\section{EFECTO DE LAS HORMONAS TIROIDEAS EN EL SISTEMA NERVIOSO CENTRAL}

Los mecanismos subyacentes a través de los cuales el funcionamiento tiroideo participa en la modulación de la psicopatología anímica y ansiosa no son del todo conocidos. Uno de los principales efectos de las hormonas tiroideas en el organismo, como es el aumento del consumo de oxígeno, no se ha demostrado en el $\mathrm{SNC}^{36}$. Por tanto, son otros los mecanismos que deben mediar la acción de estas hormonas en el SNC.

En el SNC, los receptores para T3 interactúan con una serie de genes que poseen en sus regiones regulatorias TRE (Thyroid Receptor Elements), que son los encargados de transducir la acción de la hormona. Esta acción se produce sobre la expresión génica de al menos cinco familias de proteínas ${ }^{1,37-40}$ : proteínas de mielina, neurotrofinas y sus receptores, factores de transcripción, reguladores de splicing y proteínas invo- 
lucradas en vías de señal intracelular. Estos receptores son abundantes en regiones como la amígdala e hipocampo, habiendo menos densidad de receptores en tronco y cerebelo.

Además, las hormonas tiroideas pueden actuar modulando el número de receptores alfa y beta adrenérgicos postsinápticos, tanto en corteza cerebral como en cerebelo ${ }^{41-44}$. Este hecho podńa ser relevante, considerando que la depresión seńa en parte debida a una deficiencia de catecolaminas, en particular de norepinefrina ${ }^{45,46}$. Así, en 1981 Whybrow y Prange ${ }^{47}$ plantearon la hipótesis que las hormonas tiroideas, al aumentar la función del receptor beta adrenérgico, promueven la transmisión en vías noradrenérgicas centrales y aceleran su recuperación. De esta forma, en el hipotimoidismo la disminución de receptores adrenérgicos podńa explicar la hipoactividad neuronal y con ello los efectos en la esfera anímica asociados a esta enfermedad ${ }^{46,48}$.

Un novel mecanismo de acción de T3 ha sido postulado recientemente, el cual se expresaría a través de la modulación de la concentración de serotonina intracerebral ${ }^{49,50}$. Estudios en animales han demostrado que la administración tanto aguda como crónica de T3 induce un aumento en la neurotransmisión serotoninérgica, disminuyendo la sensibilidad de autorreceptores 5- $\mathrm{HT}_{1 \mathrm{~A}}$ en el área del rafe, e incrementando la sensibilidad del receptor $5-\mathrm{HT}_{2}{ }^{51,52}$. En seres humanos, se ha podido establecer una correlación positiva entre niveles plasmáticos de serotonina y la concentración circulante de $\mathrm{T} 3^{53,54}$. Por otra parte, se ha demostrado que la serotonina cerebral disminuye en el hipotiroidismo y aumenta en el hipertiroidismo ${ }^{55-57}$. Además, la disminución de serotonina intracerebral determina un aumento en la concentración de TRH (Thyrotropin releasing hormone), que secundariamente se traduce en una elevación de hormonas tiroideas, que a su vez induce un aumento de serotonina, completándose de esta forma un mecanismo de feedback entre tiroides y SNC en lo que respecta a serotonina intracerebral (Figura 2).

De esta forma se puede especular que el efecto sobre el ánimo que se produce con la

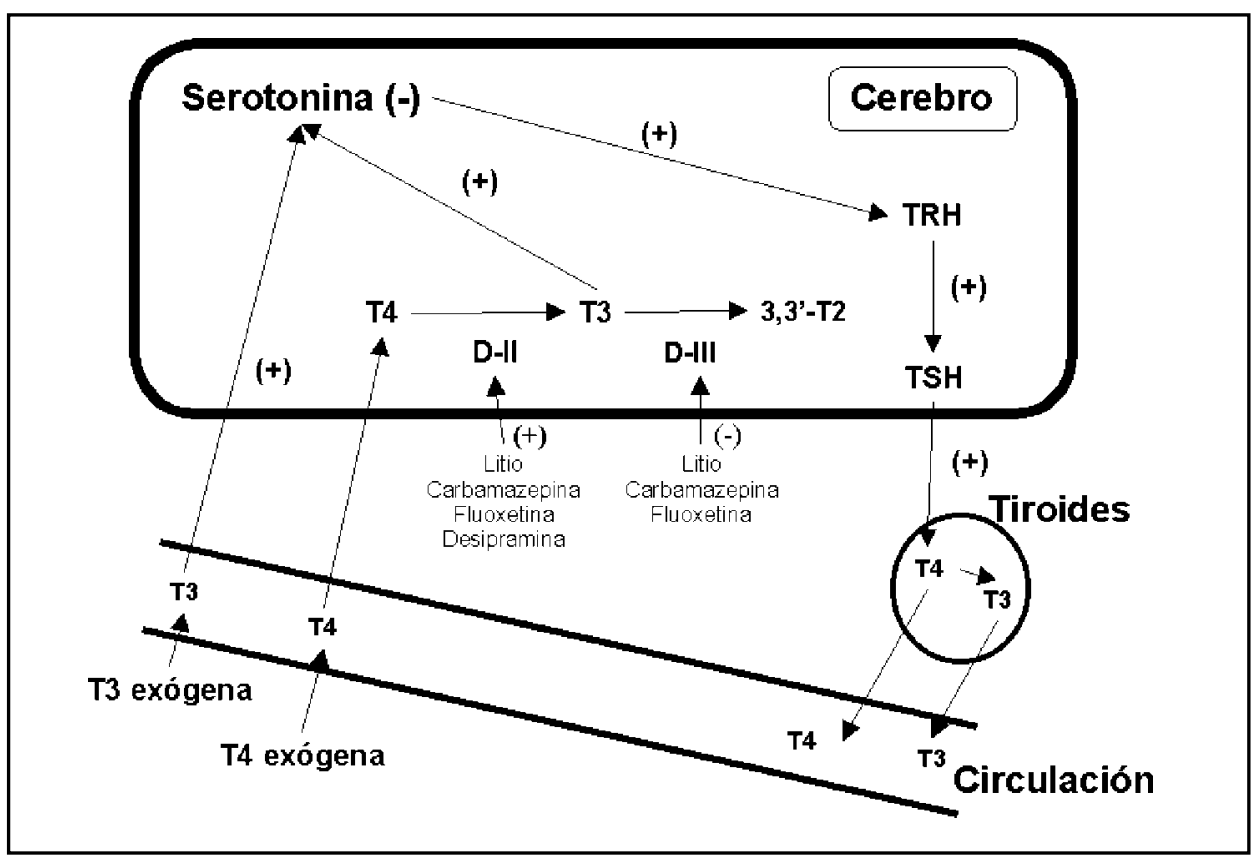

FiguRA 2. Interacción de serotonina cerebral y hormonas tiroideas. La disminución de serotonina activa el eje hipotálamo-hipófisis-tiroides e incrementa la producción de T3, que a su vez incrementa los niveles de serotonina intracerebral. Se muestran además la acción de enzimas deyodasas y los fármacos que actúan sobre ella. DII y DIII corresponde a deyodasas II y III. 3,3'-T2 es diiodotironina. (Adaptado de Kirkegaard y Faber $1998^{49}$ ), con autorización de los autores). 
administración de hormona exógena se produciría por modulación (incremento) de la neurotransmisión serotoninérgica, al igual como lo hacen diversos antidepresivos. Sin embargo, otros efectos en el postreceptor pudieran ser tan importantes como los anteriormente mencionados. Lo que es claro, es que si no hay un funcionamiento óptimo de T3 en el SNC, lo cual no necesariamente se refleja a través de la medición de hormonas periféricas, se favorecen las condiciones para la aparición de una enfermedad anímica. Apoya esta afirmación la reciente confirmación de un aumento de los niveles de T3 a nivel de la amígdala, como vía final común para el efecto de fármacos antidepresivos, estabilizadores del ánimo y deprivación de sueño ${ }^{58}$.

\section{HORMONAS TIROIDEAS Y PSICOFÁRMACOS}

Los psicofármacos y hormonas tiroideas poseen mecanismos de acción comunes, de hecho, ambos pueden modular el número de receptores postsinápticos y la concentración de serotonina intracerebral ${ }^{49,52}$, mecanismos que eventualmente podrían potenciarse. Otras interacciones se conocen por determinaciones de hormonas tiroideas antes y después del uso de psicofármacos (Tabla 2). En la mayoría de ellas se demuestra que al administrar psicofármacos, tales como fluoxetina, desipramina e inhibidores de $\mathrm{MAO}$, el efecto más constante es la disminución de los valores de T4 total y de la fracción libre de ésta ${ }^{82-85}$. Más aún, se ha postulado que los pacientes en los cuales se observa este efecto serían los que presentan mejor respuesta terapéutica ${ }^{83,85}$. La interpretación de este hecho ha sido compleja, si se considera que el efecto de hormonas tiroideas es mayor en directa relación a sus niveles tanto en plasma como en cerebro. Una explicación plausible ha sido comunicada recientemente y atribuye a los psicofármacos (antidepresivos y ciertos estabilizadores del ánimo) una acción en las enzimas deyodasa I y II, encargadas de convertir T4 en T3. Estudios en animales han demostrado que fluoxetina, desipramina, litio y carbamazepina incrementan la actividad de esta enzima y con ello la metabolización de T4 a T386-89; de este modo, los sujetos respondedores experimentarían una disminución de T4 como consecuencia de una mayor conversión a T3 (que secundariamente podría ejercer su efecto como inductor de receptores adrenérgicos, aumentar la neurotransmisión serotoninérgica 0 ambos). Más aún, también se ha objetivado que al menos litio, carbamazepina y fluoxetina disminuyen la actividad de la deyodasa III, encargada de metabolizar T3 a T2 ${ }^{86-88}$; este mecanismo tendería a aumentar aún más los niveles de T3 produciendo un efecto sinérgico con el anteriormente descrito, acrecentando el efecto del psicofármaco (Figura 2).

En contraste con el efecto benéfico que presentan algunos psicofármacos, favoreciendo la producción de T3, también es bien conocido que

Tabla 2. Efectos de fármacos sobre hormonas tiroideas

\begin{tabular}{|lccccc|}
\hline Fármaco & T3 & T4 & TSH & AC & Referencias \\
\hline Litio & $\downarrow$ & $\downarrow$ & $\uparrow$ & $+/ \uparrow$ & $(59-67)$ \\
Carbamazepina/Oxcarbamazepina & $\downarrow$ & $\downarrow$ & $\leftrightarrow$ & $\leftrightarrow /+$ & $(68-73)$ \\
Ácido valproico, Lamotrigina & $\downarrow / \leftrightarrow$ & $\downarrow / \leftrightarrow$ & $\leftrightarrow$ & $\leftrightarrow$ & $(67-72,74,75)$ \\
Gabapentina, Topiramato & $\downarrow / \leftrightarrow$ & $\downarrow / \leftrightarrow$ & $\leftrightarrow$ & $\leftrightarrow$ & $(69,71,75)$ \\
Antidepresivos tricíclicos & $\uparrow / \leftrightarrow$ & $\downarrow$ & $\leftrightarrow$ & $\leftrightarrow$ & $(76,77)$ \\
Antidepresivos ISRS & $\uparrow / \leftrightarrow$ & $\downarrow$ & $\leftrightarrow$ & $\leftrightarrow$ & $(78)$ \\
Antidepresivos IMAOs & $\leftrightarrow$ & $\leftrightarrow$ & $\leftrightarrow$ & $\leftrightarrow$ & $(79)$ \\
Antipsicóticos atípicos & $\leftrightarrow$ & $\leftrightarrow / \downarrow$ & $\leftrightarrow$ & $\leftrightarrow$ & $(80,81)$ \\
Antipsicóticos típicos & $\leftrightarrow$ & $\leftrightarrow$ & $\leftrightarrow$ & $\leftrightarrow$ & \\
Benzodiacepinas & $\leftrightarrow$ & $\leftrightarrow$ & $\leftrightarrow$ & $\leftrightarrow$ & \\
\hline
\end{tabular}

$\downarrow$ : disminuye; $\uparrow:$ aumenta; $\leftrightarrow$ : sin efecto; + : anticuerpos positivos. 
pueden tener un efecto deletéreo en la función tiroidea. La acción del litio es una de las más conocidas, ya que afectaría directamente la liberación y organificación del yodo. También puede activar la aparición de anticuerpos antitiroideos y secundariamente una tiroiditis crónica, que finalmente conduce a un hipotiroidismo. Aproximadamente $20 \%$ de los pacientes en tratamiento con litio desarrollan hipotiroidismo y este efecto se verifica generalmente durante los dos primeros años de tratamiento 62,90 .

Otro psicofármaco que también ejerce un efecto nocivo en la concentración de hormonas tiroideas es carbamazepina, al inducir enzimas hepáticas que aceleran la metabolización de hormonas tiroideas, determinando una caída en los niveles de T4 y T3, tanto total como de sus fracciones libres. Sin embargo, llama la atención que esta disminución de la concentración de hormonas tiroideas no va acompañada de un incremento paralelo de la TSH, especulándose un efecto central de carbamazepina impidiendo la elevación de esta hormona ${ }^{91,92}$. La observación clínica es que los pacientes en tratamiento con carbamazepina que presentan este efecto se benefician con el aporte de hormona tiroidea.

\section{DIRECCIONES FUTURAS DE INVESTIGACIÓN}

Existe consenso en la literatura especializada que éste constituye un campo en desarrollo donde persisten un sinnúmero de interrogantes. Dos nos parecen más relevantes y se relacionan con el metabolismo central y periférico de hormonas tiroideas, así como los fenómenos de resistencia a hormona tiroidea, tanto a nivel de receptor como postreceptor. Ambos fenómenos pueden alterar la acción de las hormonas tiroideas, sin que necesariamente exista una enfermedad en la glándula tiroides.

Metabolismo de hormonas tiroideas: Éste se encuentra controlado por la acción de las deyodasas, enzimas encargadas de convertir T4 en T3. En el ser humano se han descrito tres tipos de deyodasa, que se encuentran en distintos órganos, y en concentraciones diversas. Existen evidencias que pacientes con depresión podrían presentar una disfunción a este nivel, la cual se expresaría por niveles de T4 elevados, de T3 disminuidos, y por una elevación de rT3, tanto en plasma como en líquido céfalo-raquídeo ${ }^{49,93-95}$, concordante con un bloqueo de la deyodasa. La elevación de rT3 proviene de una metabolización alternativa de T4, que se produce al existir un bloqueo a su conversión fisiológica a T3 (Figura 1). Este bloqueo contribuiría a explicar el mejor efecto de la terapia con T3 que con T4 en pacientes depresi$\operatorname{vos}^{23}$. Algunas dudas subsisten en relación al origen de esta disfunción, ya que es un hecho conocido que la conversión periférica de hormonas tiroideas se puede afectar en una gran diversidad de condiciones, como la presencia de enfermedad orgánica debilitante, reducción de la ingesta de alimentos y uso de ciertos fármacos. Así, es posible que en episodios depresivos, esta inhibición sea secundaria a los factores que acompañan al cuadro depresivo y no relacionada primariamente con éste. Sin embargo, también podría tratarse de alteraciones primarias (genéticas o heredadas) de deficiencia de esta enzima, las cuales podrían explicar la aparición de cuadros depresivos en algunos de estos pacientes.

Resistencia a hormonas tiroideas: La resistencia a hormonas tiroideas es un síndrome caracterizado por una disminución de la respuesta de los tejidos a la acción de la hormona tiroidea ${ }^{96,97}$. La magnitud de la resistencia hormonal es dependiente del grado de alteración del receptor y generalmente es secundario a una mutación de la subunidad $\beta$ del receptor. Aun cuando la mayor parte de las resistencias son generalizadas, también se han descrito formas selectivas que comprometen sólo al receptor central (hipofisiario) o periférico, las cuales pueden expresarse clínicamente en forma diferente. Así, en las formas generalizadas de resistencia, el individuo aparece eumetabólico, con elevación de hormonas tiroideas en presencia de TSH normal. En cambio, en las formas selectivas de resistencia hipofisiaria o periférica, el paciente puede manifestar síntomas de hiper 0 hipotiroidismo. En sujetos depresivos no se ha demostrado la existencia de una resistencia genuina a hormonas tiroideas, sin embargo, existen evidencias clínicas de que pacientes depresivos particularmente refractarios podrían presentar una respuesta disminuida a la acción de hormona tiroidea (ver capítulo uso de hormonas tiroideas). Esto último estaría avalado por estudios en anima- 
les de experimentación que han demostrado que cepas de ratas «genéticamente depresivas» presentarían una disminución de la sensibilidad a hormonas tiroideas. Al confirmarse una resistencia a hormonas tiroideas en pacientes depresivos, se

\section{REFERENCIAS}

1. Yen PM. Physiological and Molecular Basis of Thyroid Hormone Action. Physiol Rev 2001; 81: 1097-142.

2. Bauer M, Szuba MP, Whybrow P. Psychiatric and Behavioral Manifestations of Hyperthyroidism and Hypothyroidism. En: Psychoneuroendocrinology: The Scientific Basis of Clinical Practice. Rothschild, AJ, American Psychiatric Publishing, Inc., 2003; 419-44.

3. Fardelia C, Gloger S, Figueroa R, Santis R, Gajardo C, Salgado C et al. High Prevalence of Thyroid Abnormalities in a Chilean Psychiatric Outpatient Population. J Endocrinol Invest 2000; 23: 102-6.

4. Prange aj J, Haggerty JJ JR, Browne JL, Rice J. Marginal Hypothyroidism in Mental Illness: Preliminary Assessment of Prevalence and Significance. En: Neuropsychopharmacology. Schmauss, M., 1990; 1: 352-61.

5. Gold MS, Pottash AL, Extein I. Hypothyroidism and Depression. Evidence from Complete Thyroid Function Evaluation. JAMA 1981; 245: 1919-22.

6. COOPER DS. Subclinical Hypothyroidism. N Engl J Med 2001; 345: 260-5.

7. TAшS F. Primary Hypothyroidism: A Case for Vigilance in the Psychological Treatment of Depression. Br J Clin Psychol 1993; 32 (Pt 3): 261-70.

8. Howland RH. Thyroid Dysfunction in Refractory Depression: Implications for Pathophysiology and Treatment. J Clin Psychiatry 1993; 54: 47-54.

9. Gloger S, Fardelia C, Santis R, Bitrán J. Relevance of the Thyroid Function Assessment in Psychiatric Patients. Rev Méd Chile 1997; 125: 1351-6.

10. Haggerty JJ JR, Prange AJ Jr. Borderline Hypothyroidism and Depression. Annu Rev Med 1995; 46: 37-46.

11. Cole DP, Thase ME, Mawnger AG, Soares JC, LUTHER JF, KUPFER DJ ET AL. Slower Treatment Response in Bipolar Depression Predicted by Lower Pretreatment Thyroid Function. Am J Psychiatry 2002; 159: 116-21. podría dar una explicación racional a la respuesta favorable que experimentan algunos pacientes con las terapias de potenciación. Además, podría justificar el uso de dosis suprafisiológicas de hormona tiroidea para alcanzar el eutiroidismo.

12. Bunevicius R, Jakubonien N, Jurkevicius R, Cernicat J, Lasas L, Prange AJ Jr. Thyroxine vs Thyroxine Plus Triiodothyronine in Treatment of Hypothyroidism after Thyroidectomy for Graves' Disease. Endocrine 2002; 18: 129-33.

13. Bunevicius R, Prange AJ. Mental Improvement after Replacement Therapy with Thyroxine Plus Triiodothyronine: Relationship to Cause of Hypothyroidism. Int J Neuropsychopharmacol 2000; 3: 167-74.

14. Sawka AM, Gerstein HC, Marriott MJ, MacQueEn GM, JofFe RT. Does a Combination Regimen of Thyroxine (T4) and 3,5,3'-Triiodothyronine Improve Depressive Symptoms Better Than T4 Alone in Patients with Hypothyroidism? Results of a Double-Blind, Randomized, Controlled Trial. J Clin Endocrinol Metab 2003; 88: 4551-5.

15. Ciyde PW, Harari AE, Getka EJ, Shakir KM. Combined Levothyroxine Plus Liothyronine Compared with Levothyroxine Alone in Primary Hypothyroidism: A Randomized Controlled Trial. JAMA 2003; 290: 2952-8.

16. Evans DL, Strawn SK, Haggerty JJ Jr, Garbutt JC, Burnett GB, Pedersen CA. Appearance of Mania in Drug-Resistant Bipolar Depressed Patients after Treatment with L-Triiodothyronine. J Clin Psychiatry 1986; 47: 521-2.

17. Whyвrow PC. The Therapeutic Use of Triiodothyronine and High Dose Thyroxine in Psychiatric Disorder. Acta Med Austriaca 1994; 21: 47-52.

18. Prange AJ J, Wilson IC, Raybon SM. Enhancement of Imipramine Antidepressant Activity by Thyroid Hormone. Am J Psychiatry 1969; 126: 457-69.

19. Wilson IC, Prange AJ J, McCiane TK. Thyroid Hormone Enhancement of Imipramine in Nonretarded Depression. N Engl J Med 1970; 282: 10637.

20. Altshuler LL, Bauer M, Frye MA, Gitun MJ, Mintz J, SzUBA MP ET AL. Does Thyroid Supplementation Accelerate Tricyclic Antidepressant Response? A Review and Meta-Analysis of the Literature. Am J Psychiatry 2001; 158: 1617-22. 
21. Altshuler LL, Frye MA, Gitun MJ. Acceleration and Augmentation Strategies for Treating Bipolar Depression. Biol Psychiatry 2003; 53: 691-700.

22. Joffe RT, Singer W, Levitt AJ, MacDonald C. A Placebo-Controlled Comparison of Lithium and Triiodothyronine Augmentation of Tricyclic Antidepressants in Unipolar Refractory Depression. Arch Gen Psychiatry 1993; 50: 387-93.

23. JoFfe RT, SINGER W. A Comparison of Triiodothyronine and Thyroxine in the Potentiation of Tricyclic Antidepressants. Psychiatry Res 1990; 32: 241-51.

24. Thase ME, KupFer DJ, JarRetT DB. Treatment of Imipramine-Resistant Recurrent Depression, I: An Open Clinical Trial Od Adjuntive L-Triiodothyronine. J Clin Psychiatry 1989; 50: 385-8.

25. Spoov J, Lahdelma L. Should Thyroid Augmentation Precede Lithium Augmentation a Pilot Study. J Affect Disord 1998; 49: 235-9.

26. Aronson R, Offman HJ, Joffe RT, Naylor CD. Triiodothyronine Augmentation in the Treatment of Refractory Depression. A Meta-Analysis. Arch Gen Psychiatry 1996; 53: 842-8.

27. Stern RA, Nevels CT, Shelhorse ME, Prohaska ML, Mason GA, Prange AJ JR. Antidepressant and Memory Effects of Combined Thyroid Hormone Treatment and Electroconvulsive Therapy: Preliminary Findings. Biol Psychiatry 1991; 30: 623-7.

28. Stern RA, Wheaun JM, Mason GA, Noonan LR, Silia SG, ARRUDA JE ET AL. Influence of L-Triiodothyronine on Memory Following Repeated Electroconvulsive Shock in Rats: Implications for Human Electroconvulsive Therapy. Biol Psychiatry 1995; 37: 198-201.

29. Rudas S, Schmitz M, Pichler P, Baumgartner A. Treatment of Refractory Chronic Depression and Dysthymia with High-Dose Thyroxine. Biol Psychiatry 1999; 45: 229-33.

30. Bauer M, Hellweg R, Graf KJ, Baumgartner A. Treatment of Refractory Depression with HighDose Thyroxine. Neuropsychopharmacology 1998; 18: 444-55.

31. Bauer MS, Whybrow PC. Rapid Cycling Bipolar Affective Disorder. II. Treatment of Refractory Rapid Cycling with High-Dose Levothyroxine: A Preliminary Study. Arch Gen Psychiatry 1990; 47: 435-40.

32. Bauer M, Priebe S, Berghofer A, Bschor T, KiessinGER U, WhyBRow PC. Subjective Response to and Tolerability of Long-Term Supraphysiological Doses of Levothyroxine in Refractory Mood Disorders. J Affect Disord 2001; 64: 35-42.
33. Gyulai L, Jaggi J, Bauer MS, Younkin S, Rubin L, Atтie M ET al. Bone Mineral Density and LThyroxine Treatment in Rapidly Cycling Bipolar Disorder. Biol Psychiatry 1997; 41: 503-6.

34. Gyulai L, Bauer M, Garcia-Espana F, Hierholzer J, Baumgartner A, Berghofer A et al. Bone Mineral Density in Pre-and Post-Menopausal Women with Affective Disorder Treated with Long-Term LThyroxine Augmentation. J Affect Disord 2001; 66: 185-91.

35. Redei EE, Solberg LC, Kluczynski JM, Pare WP. Paradoxical Hormonal and Behavioral Responses to Hypothyroid and Hyperthyroid States in the Wistar-Kyoto Rat. Neuropsychopharmacology 2001; 24: 632-9.

36. Sokoloff L, Wechsier RL, Mangold R, Balls K, Kety SS. Cerebral Blood Flow and Oxygen Consumption in Hyperthyroidism before and after Treatment. J Clin Invest 1953; 32: 202-8.

37. Farsetti A, Mitsuhashi T, Desvergne B, Robbins J, Nirodem VM. Molecular Basis of Thyroid Hormone Regulation of Myelin Basic Protein Gene Expression in Rodent Brain. J Biol Chem 1991; 266: 23226-32.

38. Farsetti A, Desvergne B, Hauenbeck P, Robbins J, NIKODEM VM. Characterization of Myelin Basic Protein Thyroid Hormone Response Element and Its Function in the Context of Native and Heterologous Promoter. J Biol Chem 1992; 267: 15784-8.

39. Strait KA, Schwartz HL, Seybold VS, Ling NC,O PPENHEIMER JH. Immunofluorescence Localization of Thyroid Hormone Receptor Protein Beta 1 and Variant Alpha 2 in Selected Tissues: Cerebellar Purkinje Cells as a Model for Beta 1 Receptor-Mediated Developmental Effects of Thyroid Hormone in Brain. Proc Natl Acad Sci USA 1991; 88: 3887-91.

40. Carlson DJ, Strait KA, Schwartz HL, Oppenheimer JH. Thyroid Hormone Receptor Isoform Content in Cultured Type 1 and Type 2 Astrocytes. Endocrinology 1996; 137: 911-7.

41. Dratman MB, Futaesaku Y, Crutchfield FL, Berman N, Payne B, Sar M et al. Iodine-125-Labeled Triiodothyronine in Rat Brain: Evidence for Localization in Discrete Neural Systems. Science 1982; 215: 309-12.

42. Dratman MB, Crutchfield FL, Axelrod J, Colburn RW, ThоA N. Localization of Triiodothyronine in Nerve Ending Fractions of Rat Brain. Proc Natl Acad Sci USA 1976; 73: 941-4. 
43. Dratman MB, CRutchfield FL. Synaptosomal [125i] Triiodothyronine after Intravenous [125i] Thyroxine. Am J Physiol 1978; 235: E638-647.

44. Joffe RT, Sokolov ST, Singer W. Thyroid Hormone Treatment of Depression. Thyroid 1995; 5: 235-9.

45. SchiLdKRAUT JJ. The Catecholamine Hypothesis of Affective Disorders: A Review of Supporting Evidence. Am J Psychiatry 1965; 122: 509-22.

46. Atterwill CK, Bunn SJ, Atrinson DJ, Smith SL, Heal DJ. Effects of Thyroid Status on Presynaptic Alpha 2-Adrenoceptor Function and Beta-Adrenoceptor Binding in the Rat Brain. J Neural Transm 1984; 59: 43-55.

47. Whybrow PC, Prange AJ Jr. A Hypothesis of Thyroid-Catecholamine-Receptor Interaction. Its Relevance to Affective Illness. Arch Gen Psychiatry 1981; 38: 106-13.

48. Bilezikian JP, Loeb JN. The Influence of Hyperthyroidism and Hypothyroidism on Alpha- and Beta-Adrenergic Receptor Systems and Adrenergic Responsiveness. Endocr Rev 1983; 4: 378-88.

49. KirkegaARd C, Faber J. The Role of Thyroid Hormones in Depression. Eur J Endocrinol 1998; 138: $1-9$.

50. Savard P, Merand Y, Di Paolo T, Dupont A. Effects of Thyroid State on Serotonin, 5-Hydroxyindoleacetic Acid and Substance P Contents in Discrete Brain Nuclei of Adult Rats. Neuroscience 1983; 10: 1399-404.

51. Sandrini M, Vitale G, Vergoni AV, Ottani A, BERTOLINI A. Effect of Acute and Chronic Treatment with Triiodothyronine on Serotonin Levels and Serotonergic Receptor Subtypes in the Rat Brain. Life Sci 1996; 58: 1551-9.

52. Bauer M, Heinz A, Whybrow PC. Thyroid Hormones, Serotonin and Mood: Of Synergy and Significance in the Adult Brain. Mol Psychiatry 2002; 7: 140-56.

53. Upadhyaya I, Agrawal JK, Dubey GP, Udupa KN. Biogenic Amines and Thyrotoxicosis. Acta Endocrinol (Copenh) 1992; 126: 315-8.

54. Cleare AJ, McGregor A, O’Keane V. Neuroendocrine Evidence for an Association between Hypothyroidism, Reduced Central 5-Ht Activity and Depression. Clin Endocrinol (Oxf) 1995; 43: 7139.

55. Singhal RL, Rastogi RB, Hrdina PD. Brain Biogenic Amines and Altered Thyroid Function. Life Sci 1975; 17: 1617-26.
56. RASTogi RB, Singhal RL. Influence of Neonatal and Adult Hyperthyroidism on Behavior and Biosynthetic Capacity for Norepinephrine, Dopamine and 5-Hydroxytryptamine in Rat Brain. J Pharmacol Exp Ther 1976; 198: 609-18.

57. ATterwill CK. Effect of Acute and Chronic TriIodothyronine (T3) Administration to Rats on Central 5-Ht and Dopamine-Mediated Behavioural Responses and Related Brain Biochemistry. Neuropharmacology 1981; 20: 131-44.

58. Pinna G, Broedel O, Eravci M, Stoltendurg-Didinger G, Plueckhan H, Fuxius S et al. Thyroid Hormones in the Rat Amygdala as Common Targets for Antidepressant Drugs, Mood Stabilizers, and Sleep Deprivation. Biological Psychiatry 2003; 54: 1049-59.

59. Bschor T, Baethge C, Adu M, Lewitzka U, Eichmann U, BAUER M. Hypothalamic-Pituitary-Thyroid System Activity During Lithium Augmentation Therapy in Patients with Unipolar Major Depression. J Psychiatry Neurosci 2003; 28: 210-16.

60. Haggerty JJ Jr, Silva SG, Marquardt M, Mason GA, Chang HY, Evans DL et al. Prevalence of Antithyroid Antibodies in Mood Disorders. Depress Anxiety 1997; 5: 91-6.

61. Rapaport MH, Schmidt ME, Risinger R, Manit $H$. The Effects of Prolonged Lithium Exposure on the Immune System of Normal Control Subjects: Serial Serum Soluble Interleukin-2 Receptor and Antithyroid Antibody Measurements. Biol Psychiatry 1994; 35: 761-6.

62. Bocchetta A, Bernardi F, Pedditzi M, Lovisew A, Veluzzzi F, Martino E et al. Thyroid Abnormalities During Lithium Treatment. Acta Psychiatr Scand 1991; 83: 193-8.

63. Bocchetta A, Bernardi F, BurRai C, Pedditzi M, Lovisew A, VelluzZi F et al. The Course of Thyroid Abnormalities During Lithium Treatment: A TwoYear Follow-up Study. Acta Psychiatr Scand 1992; 86: 38-41.

64. Haggerty JJ Jr, Evans DL, Golden RN, Pedersen CA, Simon JS, Nemeroff CB. The Presence of Antithyroid Antibodies in Patients with Affective and Nonaffective Psychiatric Disorders. Biol Psychiatry 1990; 27: 51-60.

65. Myers DH, Carter RA, Burns BH, Armond A, Hussain SB, Chengapa VK. A Prospective Study of the Effects of Lithium on Thyroid Function and on the Prevalence of Antithyroid Antibodies. Psychol Med 1985; 15: 55-61. 
66. Deniker P, Eyquem A, Bernheim R, Loo H, Delarue P. Thyroid Autoantibody Levels During Lithium Therapy. Neuropsychobiology 1978; 4: 270-5.

67. Barclay ML, Brownle BE, Turner JG, Wells JE. Lithium Associated Thyrotoxicosis: A Report of 14 Cases, with Statistical Analysis of Incidence. Clin Endocrinol (Oxf) 1994; 40: 759-64.

68. YuKSEL A, YaLCIN E, CENANI A. Influence of LongTerm Carbamazepine Treatment on Thyroid Function. Acta Paediatr Jpn 1993; 35: 229-32.

69. Isojarvi JI, Pakarinen AJ, Myшyla VV. Thyroid Function with Antiepileptic Drugs. Epilepsia 1992; 33: 142-8.

70. Eiris-Punal J, Del Río-Garma M, Del Río-Garma MC, Lojo-Rocamonde S, Novo-Rodríguez I, CaSTro-Gago M. Long-Term Treatment of Children with Epilepsy with Valproate or Carbamazepine May Cause Subclinical Hypothyroidism. Epilepsia 1999; 40: 1761-6.

71. Zhu SQ, Lu XM, Ruan XZ, Cai Z. Changes of Thyroid Hormone Levels in Epileptic Patients. J Tongji Med Univ 1994; 14: 119-23.

72. Isojarvi JiT, Turkka J, Pakarinen AJ, Kotila M, Rattya J, Myцyla VV. Thyroid Function in Men Taking Carbamazepine, Oxcarbazepine, or Valproate for Epilepsy. Epilepsia 2001; 42: 930-4.

73. Premachandra BN, Radparvar A, Burman $K$, Wiwhams IK. Apparent Increase in Type I 5'-Deiodinase Activity Induced by Antiepileptic Medication in Mentally Retarded Subjects. Horm Res 2002; 58: 273-8.

74. Ericsson UB, Bjerre I, Forsgren M, Ivarsson SA. Thyroglobulin and Thyroid Hormones in Patients on Long-Term Treatment with Phenytoin, Carbamazepine, and Valproic Acid. Epilepsia 1985; 26: 594-6.

75. Larkin JG, Macphee GJ, Beastall GH, Brodie MJ. Thyroid Hormone Concentrations in Epileptic Patients. Eur J Clin Pharmacol 1989; 36: 213-6.

76. Brady KT, Lydiard RB, Keluner CH, Joffe R, Laird LK, Morton WA et AL. A Comparison of the Effects of Imipramine and Fluvoxamine on the Thyroid Axis. Biol Psychiatry 1994; 36: 778-9.

77. Brady KT, Anton RF. The Thyroid Axis and Desipramine Treatment in Depression. Biol Psychiatry 1989; 25: 703-9.

78. Shelton RC, Winn S, Ekhatore N, Loosen PT. The Effects of Antidepressants on the Thyroid Axis in Depression. Biol Psychiatry 1993; 33: 120-6.
79. JofFe RT, Singer W. Effect of Phenelzine on Thyroid Function in Depressed Patients. Biol Psychiatry 1987; 22: 1033-5.

80. Feret BM, Caley CF. Possible Hypothyroidism Associated with Quetiapine. Ann Pharmacother 2000; 34: 483-6.

81. McManus DQ, Arvanitis LA, Kowalcyk BB. Quetiapine, a Novel Antipsychotic: Experience in Elderly Patients with Psychotic Disorders. Seroquel Trial 48 Study Group. J Clin Psychiatry 1999; 60: 292-8.

82. Duval F, Mokrani MC, Croco MA, Jautz M, Bailey P, DieP TS ET AL. Effect of Antidepressant Medication on Morning and Evening Thyroid Function Tests During a Major Depressive Episode. Arch Gen Psychiatry 1996; 53: 833-40.

83. Sokolov ST, KuTcher SP, JofFe RT. Changes in Thyroid Hormone Levels Associated with Desipramine Response in Adolescent Depression. Prog Neuropsychopharmacol Biol Psychiatry 1996; 20: 1053-63.

84. JoFFe RT, SINGER W. Antidepressants and Thyroid Hormone Levels. Acta Med Austriaca 1992; 19 Suppl 1: 96-7.

85. Kusalic M, Engelsmann F, Bradwejn J. Thyroid Functioning During Treatment for Depression. J Psychiatry Neurosci 1993; 18: 260-3.

86. Baumgartner A, Campos-Barros A, Gaio U, HesseNiUs C, Frege I, Meinhold H. Effects of Lithium on Thyroid Hormone Metabolism in Rat Frontal Cortex. Biol Psychiatry 1994; 36: 771-4.

87. Baumgartner A, Campos-Barros A, Gaio U, Hessenius C, Flechner A, Meinhold H. Carbamazepine Affects Triiodothyronine Production and Metabolization in Rat Hippocampus. Life Sci 1994; 54: PL401-407.

88. Baumgartner A, Dubeyko M, Campos-Barros A, ERAVCi M, MeinHold H. Subchronic Administration of Fluoxetine to Rats Affects Triiodothyronine Production and Deiodination in Regions of the Cortex and in the Limbic Forebrain. Brain Res 1994; 635: 68-74.

89. Campos-Barros A, Meinhold H, Stula M, Muler F, KoHler R, ERAVCI M ET aL. The Influence of Desipramine on Thyroid Hormone Metabolism in Rat Brain. J Pharmacol Exp Ther 1994; 268: 114352.

90. BARSANO CP. Other Forms of Primary Hypothyroidism. En: The Thyroid. Utigerm R, LippincotRaven, 1996; 771-2. 
91. Roy-Byrne PP, JofFe RT, Uhde TW. Carbamazepine and Thyroid Function in Affective Il Patient. Clinical and Theoretical Implications. Arch Gen Psychiatry 1984; 41: 1150-3.

92. Wenzel KW. Disturbances of Thyroid Function Tests by Drugs. Acta Med Austriaca 1996; 23: 57-60.

93. Baumgartner A, Graf KJ, Kurten I, Meinhold H. The Hypothalamic-Pituitary-Thyroid Axis in Psychiatric Patients and Healthy Subjects: Parts 14. Psychiatry Res 1988; 24: 271-332.
94. KirKegaARD C, Faber J. Free Thyroxine and 3,3',5'Triiodothyronine Levels in Cerebrospinal Fluid in Patients with Endogenous Depression. Acta Endocrinol (Copenh) 1991; 124: 166-72.

95. KIRKEGAARD C, FABER J. Influence of Free Thyroid Hormone Levels on the TSH Response to TRH in Endogenous Depression. Psychoneuroendocrinology 1986; 11: 491-7.

96. ReFETofF S. Resistance to Thyroid Hormone. Curr Ther Endocrinol Metab 1997; 6: 132-4.

97. Weiss RE, ReFetoff S. Resistance to Thyroid Hormone. Rev Endocr Metab Disord 2000; 1: 97-108. 